\title{
Efecto del número de inseminaciones y de la GnRH sobre la preñez en vaquillonas Angus sincronizadas con tiaprost*
}

\author{
Callejas, S.; Larghi, J.; Zeberio, M.; Catalano, R.; Cabodevila, J. \\ Área de Reproducción, Facultad de Ciencias Veterinarias, Universidad Nacional del Centro de la Provincia de \\ Buenos Aires, Campus Universitario (B7000GHG), Tandil, Prov. Buenos Aires, Argentina. \\ Tel. 02293-439850, E-mail: callejas@vet.unicen.edu.ar.
}

\begin{abstract}
Resumen
Callejas, S.; Larghi, J.; Zeberio, M.; Catalano, R.; Cabodevila, J.: Efecto del número de inseminaciones y de la GnRH sobre la preñez en vaquillonas Angus sincronizadas con tiaprost. Rev. vet. 20: 1, $22-24,2009$. Los objetivos del trabajo fueron evaluar el efecto del número de inseminaciones (inseminación artificial a tiempo fijo: IATF, 2 vs. 1) en vaquillonas sincronizadas con dos inyecciones de un agente luteolítico PGF (tiaprost) separadas por 12 días y comprobar si la administración de $\mathrm{GnRH}$ al realizar una IATF permite obtener resultados de preñez equivalentes a dos IATF. Se realizaron tres réplicas del ensayo, utilizando 98 vaquillonas Angus (edad: 18-24 meses; condición corporal: 7,2 $\pm 0,4$ - escala 1 a 9). Las vaquillonas fueron distribuidas aleatoriamente dentro de cada repetición. 1) Grupo PGF2IATF: en los días 0 y 12 se administraron $750 \mu \mathrm{g}$ de tiaprost, realizando IATF a las 72 y $96 \mathrm{~h}$ post PGF. 2) Grupo PGFGnRHIATF: ídem grupo PGF2IATF, realizando una IATF a las $72 \mathrm{~h}$ y administración de $8 \mu \mathrm{g}$ de buserelina. 3) Grupo PGFIATF: similar al grupo anterior, pero sin buserelina. Para la IATF se utilizó semen congelado / descongelado, proveniente de un toro de probada fertilidad. El diagnóstico de gestación se realizó por palpación transrrectal a los 90 días post IATF. El análisis estadístico se realizó con el auxilio del SAS, sin observar efecto de la repetición $(\mathrm{p}>0,05)$. Los animales del grupo PGFIATF se preñaron en menor proporción que los inseminados a las 72 y 96 horas (46,9 vs. 76,5\%, respectivamente). El grupo que incluyó buserelina (GnRH) no mostró diferencias con los otros grupos $(59,4 \%, p>0,05)$. Se concluye que en vaquillonas Angus sincronizadas con dos inyecciones de PGF separadas por 12 días, la implementación de una IATF realizada a las 72 horas post tratamiento disminuye el porcentaje de preñez comparado con dos IATF a las 72 y 96 horas. El uso de GnRH evita tal disminución, circunstancia que debería ser confirmada sobre un mayor número de animales.
\end{abstract}

Palabras clave: vaquillona, inseminación a tiempo fijo, agente luteolítico, preñez.

\begin{abstract}
Callejas, S.; Larghi, J.; Zeberio, M.; Catalano, R.; Cabodevila, J.: Effect of number of insemination and GnRH administration on pregnancy rate in beef heifers treated with luteolytic agent. Rev. vet. 20: 1, $22-24,2009$. This study was carried out to evaluate the effect of insemination number (timed artificial insemination: TAI, 2 versus 1) on pregnancy rate in beef heifers treated with two injections of a luteolytic agent (PGF, tiaprost), 12 days between each administration, and to evaluate whether if $\mathrm{GnRH}$ administration at one TAI produces similar pregnancy rate compared to two TAI. The experiment was repeated three times, using 98 Angus heifers (18-24 months old, body condition score: $7.2 \pm 0.4$, scale 1-9). Heifers were distributed randomly within each repetition, as follows: 1) Group PGF2TAI: on days 0 and 12, animals were administered with $750 \mu \mathrm{g}$ tiaprost and received two TAI, 72 and $96 \mathrm{~h}$ after PGF. 2) Group PGFGnRHTAI: similar to previous treatment, but received one TAI $72 \mathrm{~h}$ after PGF and $8 \mu \mathrm{g}$ of busereline. 3) Group PGFTAI: similar to previous group, but without busereline. A bull of proven fertility was used for the TAI. Pregnancy detection was performed using rectal palpation 90 days after TAI. SAS was used for the statistical analysis; effect of the repetition was not observed ( $p>0.05)$. Pregnancy rate was lower in animals that received TAI $72 \mathrm{~h}$ after PGF without GnRH $(46.9 \%)(\mathrm{p}<0.05)$, compared to those that receive two TAI 72 and $96 \mathrm{~h}$ after PGF (76.5\%). Group with GnRH did not show significant diffe-
\end{abstract}

Recibido: 4 diciembre 2008 / Aceptado: 3 febrero 2009

* Proyecto "Aumento de la eficiencia de técnicas aplicadas a la reproducción en rumiantes" (Área de Reproducción, FISFARVET, UNCPBA). Resumen presentado en el $31^{\circ}$ Congreso Argentino de Producción Animal, Potrero de los Funes (San Luís, Argentina), octubre de 2008. 
rences compared to the others $(59.4 \%, \mathrm{p}>0.05)$. It can be concluded that, for Angus heifers treated with two injections of PGF 12 days between each administration, the implementation of one TAI 72 hours after PGF diminishes pregnancy rate compared to two TAI (72 and 96 h). The use of the GnRH avoids such decrease.

Key words: heifer, timed artificial insemination, luteolytic agent, pregnancy.

\section{INTRODUCCIÓN}

El control farmacológico del ciclo permite incorporar más fácilmente la inseminación artificial (IA), ya que en un periodo corto se puede inseminar a la mayor parte del rodeo. Además, existen protocolos que permiten efectuar IA a tiempo fijo (IATF) sin necesidad de realizar detección de celos, ya que el tratamiento permite sincronizar el momento en que ocurren las ovulaciones. Es sabido que los problemas en la detección de celos son los principales obstáculos que limitan el uso de la IA ${ }^{3}$.

Los tratamientos utilizados para controlar el momento de la ovulación y realizar IATF se basan en el uso de agentes luteolíticos (prostaglandinas F, PGF), como el protocolo que utiliza dos inyecciones separadas por 12 días ${ }^{5}$, o aquellos esquemas que incluyen como droga base a la progesterona (ej. dispositivos intravaginales que liberan dicha hormona y permanecen colocados durante 7-8 días) ${ }^{7,8}$.

El uso de dos inyecciones de PGF ha sido ampliamente estudiado en la década de 1970, con resultados satisfactorios cuando se realizan dos IATF, una a las 72 y otra a las 96 horas de administrada la segunda PGF $^{5}$. La reducción en el número de IATF a una sola a la hora 72 , ha mostrado resultados variables ${ }^{6}$; así, en vaquillonas lecheras el porcentaje disminuyó significativamente $(p=0,01)$, de 57,1 a $40,6 \%$ y en vaquillonas de cría, la disminución fue de 49,1 a 37,8\% ( $\mathrm{p}=0,001)$. No obstante, en algunos ensayos se han obtenido muy buenas tasas de preñez y de parición al realizar una sola IATF (67 y $61 \%$, respectivamente) ${ }^{6}$. Por otro lado, la buserelina (GnRH) se ha utilizado para controlar el momento en que se produce la ovulación en animales que reciben el tratamiento OvSynch (GnRH día 0, PGF día 7 y GnRH día 9), pudiendo implementarse una sola IATF.

En consecuencia, los objetivos del presente trabajo fueron evaluar el efecto del número de inseminaciones (2 vs. 1) en vaquillonas sincronizadas con dos dosis de un agente luteolítico PGF (tiaprost) separadas por 12 días y comprobar si el uso de GnRH administrada en el momento de realizar la IATF a la hora 72 permite mejorar la tasa de preñez que se obtiene al realizar una sola IATF.

\section{MATERIAL Y MÉTODOS}

Lugar: el ensayo se realizó en un establecimiento rural ubicado en el partido de Tandil, Provincia de Buenos Aires.
Animales: se realizaron 3 réplicas del ensayo (I, II y III), utilizando 98 vaquillonas Angus negras y coloradas de 18-24 meses de edad y condición corporal de $7,2 \pm 0,4$ (escala 1-9; 1: excesivamente flaca y 9: excesivamente gorda). El $82,7 \%$ de las vaquillonas poseía cuerpo lúteo, determinado por palpación transrrectal.

Tratamientos: las vaquillonas fueron distribuidas aleatoriamente dentro de cada repetición, a tres grupos que recibieron los siguientes tratamientos:

1. Grupo PGF2IATF: en los días 0 y 12 se administraron por vía intramuscular $50 \mu \mathrm{g}$ de tiaprost (Iliren ${ }^{\circledR}$, Intervet) en cada aplicación, realizando IATF a las 72 y $96 \mathrm{~h}$ posteriores a la segunda inyección.

2. Grupo PGFGnRHIATF: idem al tratamiento anterior, pero se realizó una sola IATF a las $72 \mathrm{~h}$ y en ese momento se administraron por vía intramuscular $8 \mu \mathrm{g}$ de GnRH (buserelina: Receptal ${ }^{\circledR}$, Intervet).

3. Grupo PGFIATF: similar al grupo anterior, pero sin administrar buserelina.

Servicio: se realizó por IATF, utilizando semen congelado/ descongelado en pajuelas de $0,5 \mathrm{ml}$, provenientes de un toro de probada fertilidad.

Diagnóstico de gestación: se efectuó por palpación transrrectal a los 90 días de realizada la IATF.

Análisis estadístico: se evaluó el efecto del tratamiento y de la repetición sobre el porcentaje de preñez a la IATF. Se utilizó el subprograma CATMOD, perteneciente al paquete estadístico SAS, fijando un nivel de confianza del $95 \%(\alpha=0,05)$.

\section{RESULTADOS}

Se observó un efecto significativo del tratamiento $(\mathrm{p}<0,05)$; no así de la repetición ( $\mathrm{p}>0,05)$. Los animales que fueron inseminados a tiempo fijo a la hora 72 y no recibieron GnRH se preñaron en menor proporción que aquellos que fueron inseminados a las 72 y 96 horas, mientras que el grupo que recibió GnRH no mostró diferencias significativas con los grupos antes mencionados (Tabla 1).

\section{DISCUSIÓN}

El porcentaje de preñez registrado en el grupo que recibió dos IATF se ubicó por encima del rango citado en la bibliografía, que varía de 49 a 70\% (revisado por Callejas ${ }^{2}$ ). Cabe señalar que las vaquillonas se encontraban en buena condición corporal y ciclando $(82,7 \%$ de vaquillonas con cuerpo lúteo), lo que explicaría la respuesta observada en este grupo. 
Tabla 1. Preñez en vaquillonas Angus con tres sistemas de inseminación a tiempo fijo.

\begin{tabular}{cccc}
\hline repetición & tratamiento & \multicolumn{2}{c}{ preñez* $(\%)$} \\
\hline \multirow{2}{*}{ I } & PGF2IATF & $63,6(7 / 11)$ & $48,4 \mathrm{a}$ \\
& PGFGnRHIATF & $40,0(4 / 10)$ & $(15 / 31)$ \\
& PGFIATF & $40,0(4 / 10)$ & \\
\hline \multirow{3}{*}{ II } & PGF2IATF & $75,0(6 / 8)$ & $66,7 \mathrm{a}$ \\
& PGFGnRHIATF & $75,0(6 / 8)$ & $(16 / 24)$ \\
& PGFIATF & $50,0(4 / 8)$ & \\
\hline \multirow{3}{*}{ III } & PGF2IATF & $86,7(13 / 15)$ & $67,4 \mathrm{a}$ \\
& PGFGnHIATF & $64,2(9 / 14)$ & $(29 / 43)$ \\
& PGFIATF & $50,0(7 / 14)$ & \\
\multirow{3}{*}{ I+II+III } & PGFFIIATF & $76,5 \mathrm{a}(26 / 34)$ & 61,2 \\
& PGFIATF & $46,9 \mathrm{~b}(15 / 32)$ & $(60 / 98)$ \\
\hline
\end{tabular}

*Valores en una misma columna con superíndices diferentes, difieren: $\mathrm{p}<0,05$.

Por otro lado, en coincidencia con lo informado por otro investigador ${ }^{6}$, la reducción en el número de inseminaciones ( 2 a 1), afectó negativamente el porcentaje de preñez. En consecuencia, este hecho debe ser tenido en cuenta a la hora de implementar este tipo de protocolo de control del ciclo estral, ya que se podría afectar negativamente la eficiencia económica del sistema.

En otro ensayo se observó un bajo porcentaje de preñez (25\%) luego de realizar una IATF en vaquillonas Holando Argentino, cíclicas, sincronizadas con dos dosis de PGF separadas por 11 días, registrándose una dispersión de celos importante, lo que podría indicar que una sola IATF no fue lo más adecuado ${ }^{1}$. Por otro lado, estos investigadores utilizaron el método Ovsynch (GnRH-PGF-GnRH) en otro grupo de vaquillonas, obteniendo porcentajes de preñez del $66,7 \%$. Esto sería consecuencia de que la GnRH sincronizó la ovulación y permitió que luego de realizada la IATF se logren los resultados observados.

La GnRH es un decapéptido de origen hipotalámico cuya función es estimular la liberación de las hormonas folículoestimulante (FSH) y luteinizante (LH) producidas en la hipófisis. La administración de GnRH en hembras bovinas induce un aumento en los niveles plasmáticos de LH y FSH por un período de $4 \mathrm{a} 6$ horas $4,9,10$ con lo cual ejerce en forma indirecta su acción sobre las estructuras ováricas. En el presente trabajo, la GnRH podría haber inducido una mejor sincronía en la ovulación de las hembras tratadas, evitando así la disminución en el porcentaje de preñez observado en aquellas vaquillonas que no la recibieron y fueron inseminadas en una sola oportunidad.

En otros trabajos, la GnRH también fue utilizada en el momento de la IATF con el objetivo de sincronizar la ovulación en animales que habían recibido otros tratamientos de control del ciclo estral, como aquellos basados en el uso de progesterona ${ }^{7,8}$ con buenos resul- tados, equivalentes a aquellos que utilizan benzoato de estradiol, para cumplir tal objetivo.

Se concluye que en vaquillonas Angus sincronizadas con el protocolo que utiliza dos inyecciones de un agente luteolítico separadas por 12 días, la implementación de una sola IATF realizada a las 72 horas post tratamiento disminuye el porcentaje de preñez comparado con la realización de dos IATF a las 72 y 96 horas. El uso de GnRH administrada en el momento de realizar la IATF evita tal disminución. No obstante, los resultados observados en el presente trabajo deberían ser confirmados sobre un mayor número de animales.

\section{REFERENCIAS}

1. Callejas S, Ersinger C, Cabodevila J, Catalano R, Teruel M, Calá M. 1999. Uso de la hormona liberadora de gonadotrofinas y de la prostaglandina F2 $\alpha$ para realizar una inseminación artificial sistemática. Taurus 2: 4-8.

2. Callejas S. 2004. Control farmacológico del ciclo estral bovino: bases fisiológicas, protocolos y resultados. Parte I. Taurus 24: 22-34.

3. Catalano C, Callejas S. 2001. Detección de celos en bovinos. Factores que la afectan y métodos de ayuda. Rev Med Vet 82: 17-22.

4. Chenault JR, Kratzer DD, Rzepkowski RA, Godwin MC. 1990. LH and FSH response of Holstein heifers to fertirelin acetate, gonadorelin and buserelin. Theriogenology 34: 81-98.

5. Cooper MJ. 1974. Control of oestrous cycles of heifers with a synthetic prostaglandin analogue. Vet Rec 95: 200-203.

6. Cooper MJ. 1976. The use of cloprostenol ("Estrumate") in the controlled breeding of cattle. An assessment of European field trials. Proceeding of a Symposium Oestrus Synchronization in Cattle, Sydney (Australia), p. 24-38.

7. De Dominici O, Callejas S. 2004. Fertilidad de la IATF realizada después de la administración de benzoato de estradiol o de GnRH al final de un tratamiento con progesterona/progestágenos en vacas para carne. Rev Arg Prod Anim 24 (Supl. 1): 280-281.

8. Huguenine E, Cledou G, Callejas S. 2008. IATF en vacas secas: Uso de dispositivos intravaginales con progesterona combinados con GnRH o benzoato de estradiol. Anales XXXIV Jornadas Uruguayas de Buiatría (Paysandú, Uruguay), p. 160-161.

9. Mee MO, Stevenson JS, Alexander BM, Garth Sasser R. 1993. Administration of GnRH at estrus influences pregnancy rates, serum concentrations of LH, FSH, estradiol$17 \beta$, Pregnancy-Specific Protein B, and progesterone, proportion of luteal cell types, and in vitro production of progesterone in dairy cows. J Anim Sci 71: 185-198.

10. Rosenberg M, Chun S, Kaim M, Herz Z, Folman Y. 1991. The effect of GnRH administered to dairy cows during oestrus on plasma LH and conception in relation to the time of treatment and insemination. Anim Reprod Sci 24 : $13-24$. 